\title{
Extracting observables from lattice data in the three-particle sec- tor
}

\author{
Akaki Rusetsky ${ }^{1, \star}$, Hans-Werner Hammer ${ }^{2}$, and Jin-Yi Pang ${ }^{1}$ \\ ${ }^{1}$ Helmholtz-Institut für Strahlen- und Kernphysik and Bethe Center for Theoretical Physics, \\ University of Bonn, Nussallee 14-16, 53115 Bonn, Germany \\ ${ }^{2}$ Institut für Kernphysik, Technische Universität Darmstadt, 64289 Darmstadt, Germany and \\ ExtreMe Matter Institute EMMI, GSI Helmholtzzentrum für Schwerionenforschung, \\ 64291 Darmstadt, Germany
}

\begin{abstract}
The three-particle quantization condition is derived, using the particle-dimer picture in the non-relativistic effective field theory. The procedure for the extraction of various observables in the three-particle sector (the particle-dimer scattering amplitudes, breakup amplitudes, etc.) from the finite-volume lattice spectrum is discussed in detail. As an illustration of the general formalism, the expression for the finite-volume energy shift of the three-body bound-state in the unitary limit is re-derived. The role of the threebody force, which is essential for the renormalization, is highlighted, and the extension of the result beyond the unitary limit is studied. Comparison with other approaches, known in the literature, is carried out.
\end{abstract}

\section{Introduction}

Recent years have seen an increased interest in the calculation of the scattering observables through lattice simulations. Whereas in the two-body sector this meanwhile became a pretty standard procedure based on the so-called Lüscher method [1] (for the extraction of the scattering phases) and the Lellouch-Lüscher formula [2] (for the extraction of the matrix elements), no comparable framework exists in the three-body sector so far, despite some activity in this field during the last few years [3-18]. In particular, in Ref. [8] a three-particle quantization condition has been derived, which determines the finite-volume spectrum in the three-particle sector in terms of the infinite-volume quantities. However, this condition has a rather complicated form and contains unconventional scattering matrix elements that explicitly depend on the introduced cutoff. For this reason, the use of this condition in the analysis of the real lattice data could be difficult.

Recently, in Refs. [19, 20] we have proposed a simpler formalism, which is based on the reformulation of the three-particle problem within the particle-dimer picture. It has been shown that this formalism, within its range of applicability, is equivalent to the quantization condition derived in Ref. [8], but is much simpler in the applications. This is achieved mainly by choosing a convenient set of parameters to be fit to the lattice data. Namely, the particle-dimer effective Lagrangian contains a tower of the low-energy couplings of increasing orders that correspond to the three-body short-range

${ }^{\star}$ Speaker, e-mail: rusetsky@hiskp.uni-bonn.de 
interactions in the three-particle picture. In our approach, these couplings represent the only unknown quantities, which determine the three-particle dynamics both in a finite and in the infinite volume and which should be fitted to the lattice data (we remind the reader that the effective couplings in the two-particle sector can be determined separately from the two-particle simulations by using the conventional Lüscher approach). Thus, in the energy region, where the particle-dimer interactions could be considered local, the parameterization of this interaction in terms of the first few effective couplings is justified and the lattice data on the finite-volume energy spectrum fully determine the three-particle dynamics in the infinite volume.

In this work we briefly review the formalism of Refs. [19, 20]. As an illustration of the general method, we consider the calculation of the finite-volume energy shift of a three-particle shallow bound state and demonstrate that our approach allows one to go beyond the unitary limit considered in Refs. $[4,12]$. Further, we clarify the role of the three-body force in these calculations.

\section{The formalism}

In our derivation, in order to keep notations as simple as possible, we restrict ourselves to the case of the non-relativistic identical particles. Including the above effects does not change the essence of the method and can be carried out straightforwardly.

The two- and three-particle interactions in the non-relativistic effective field theory (NREFT) are described by the Lagrangian

$$
\mathcal{L}=\psi^{\dagger}\left(i \partial_{0}+\frac{\nabla^{2}}{2 m}\right) \psi+\mathcal{L}_{2}+\mathcal{L}_{3}
$$

where $\psi(x)$ denotes a non-relativistic field, $m$ is the mass of the particle, and $\mathcal{L}_{2}, \mathcal{L}_{3}$ denote the twoand three-particle interaction terms, respectively. For example,

$$
\mathcal{L}_{2}=-\frac{C_{0}}{2} \psi^{\dagger} \psi^{\dagger} \psi \psi+\frac{C_{2}}{4}\left(\psi^{\dagger} \stackrel{\leftrightarrow}{\nabla}{ }^{2} \psi^{\dagger} \psi \psi+\text { h.c. }\right)+O\left(\nabla^{4}\right)
$$

Here, the first term corresponds to a non-derivative interaction which is purely S-wave. In the second term, we employ the Galilean invariant derivative operator $\stackrel{\leftrightarrow}{\nabla} \equiv(\vec{\nabla}-\stackrel{\leftarrow}{\nabla}) / 2$, which is understood to act only on the fields immediately left and right of the operator.

The three-particle term in the CM frame of three particles is given by

$$
\mathcal{L}_{3}=-\frac{D_{0}}{6} \psi^{\dagger} \psi^{\dagger} \psi^{\dagger} \psi \psi \psi-\frac{D_{2}}{12}\left(\psi^{\dagger} \psi^{\dagger} \nabla^{2} \psi^{\dagger} \psi \psi \psi+\text { h.c. }\right)+O\left(\nabla^{4}\right) \text {. }
$$

Here, again, the derivative acts only on the field immediately right of the operator $\nabla^{2}$. The effective couplings $C_{i}, D_{i}$ should be determined - order by order - through the matching of the calculated $S$ matrix elements to the low-energy observables in the two-particle sector. In particular, the couplings $C_{i}$ are directly related to the effective-range expansion parameters in the two-body scattering - the scattering length $a$, the effective radius $r$, and so on. Note that, in order to reduce the number of the independent couplings, one should systematically use the equations of motion. It can be rigorously shown that the terms, which are eliminated in this manner, do not contribute to the $S$-matrix. In the context of the present problem, we demonstrate that exactly the same technique can be used to treat the Green functions in a finite volume. Therefore, the terms that are eliminated with the use of the equations of motion, do not contribute to the finite-volume spectrum and hence the latter is fully determined by the on-shell $S$-matrix in the infinite volume. This statement has been first proved in Ref. [3] and was confirmed in Ref. [8]. 
Next, we note that the use of the particle-dimer language in the context of the three-particle problem grossly simplifies the framework in the infinite volume and the same is expected in a finite volume as well. The transition to the particle-dimer picture can be accomplished by introducing auxiliary integration variables in the path integral that defines the Green functions in the three-particle theory. In this sense, the particle-dimer picture is not an approximation, but an equivalent description of the same problem. The effective Lagrangian in the particle-dimer picture takes the form

$$
\begin{aligned}
\mathcal{L} & =\psi^{\dagger}\left(i \partial_{0}+\frac{\nabla^{2}}{2 m}\right) \psi+\sigma T^{\dagger} T+\left(T^{\dagger} \psi[f(-i \nabla)] \psi+\text { h.c. }\right) \\
& +h_{0} T^{\dagger} T \psi^{\dagger} \psi+h_{2} T^{\dagger} T\left(\psi^{\dagger} \nabla^{2} \psi+\nabla^{2} \psi^{\dagger} \psi\right) \\
& +h_{4} T^{\dagger} T\left(\psi^{\dagger} \nabla^{4} \psi+\nabla^{4} \psi^{\dagger} \psi\right)+h_{4}^{\prime} T^{\dagger} T \nabla^{2} \psi^{\dagger} \nabla^{2} \psi+\cdots,
\end{aligned}
$$

where

$$
\begin{aligned}
\psi[f(-i \nabla)] \psi & =f_{0} \psi \psi+f_{1} \psi(-i \stackrel{\leftrightarrow}{\nabla})^{2} \psi+f_{2} \psi(-i \stackrel{\leftrightarrow}{\nabla})^{4} \psi+\cdots, \\
\psi(-i \stackrel{\leftrightarrow}{\nabla})^{2} \psi & =-\psi \nabla^{2} \psi+\nabla_{i} \psi \nabla_{i} \psi, \quad \text { and so on. }
\end{aligned}
$$

In the above equations, $T$ denotes the (scalar) dimer field (for simplicity, we have restricted ourselves only to the S-wave in pair interactions, but the higher partial waves can be systematically taken into account by introducing a tower of dimers with different spins), $\sigma= \pm 1$ ( $\sigma=-1$ if $C_{0}>0$ and vice versa) and the couplings $f_{0}, f_{1}, \ldots$ and $h_{0}, h_{2}, \ldots$ can be systematically matched to the couplings $C_{i}, D_{i}$ in perturbation theory (the explicit form of the matching condition depends on the regularization used). As stated above, the particle-dimer picture is completely equivalent to the tree-particle picture integration out the auxiliary field $T$ in the path integral, we arrive at the Lagrangian given in Eqs. (1), (2), (3) ${ }^{1}$. Further, note that, in the particle-dimer picture, the off-shell terms in the three-particle sector, which vanish through the use of the equations of motion, do not emerge from the beginning. The reason for this is simple: since the dimer is an auxiliary field, it does not have a fixed mass. Hence, the kinematical constraints allow to relate the particle-dimer scattering amplitude for arbitrary external momenta off energy shell to the three-particle scattering amplitude with the on-shell particles. Consequently, all couplings, emerging in the particle-dimer interaction Lagrangian, are physical and cannot be eliminated with the use of the equations of motion.

The particle-dimer scattering amplitude obeys the Bethe-Salpeter integral equation. In the simplest case of the spin-0 dimer and of only non-derivative interactions, the equation takes the following form

$$
\mathcal{M}(\mathbf{p}, \mathbf{q} ; E)=Z(\mathbf{p}, \mathbf{q} ; E)+8 \pi \int^{\Lambda} \frac{d^{3} \mathbf{k}}{(2 \pi)^{3}} Z(\mathbf{p}, \mathbf{k} ; E) \tau(\mathbf{k} ; E) \mathcal{M}(\mathbf{k}, \mathbf{q} ; E) .
$$

Here, $E$ denotes the total CM energy of three particles, $a$ is the S-wave scattering length, and

$$
\begin{aligned}
Z(\mathbf{p}, \mathbf{q} ; E) & =\frac{1}{-m E+\mathbf{p}^{2}+\mathbf{q}^{2}+\mathbf{p q}}+\frac{h_{0}}{m f_{0}^{2}}, \\
\tau(\mathbf{k} ; E) & =\frac{1}{-a^{-1}+\sqrt{\frac{3}{4} \mathbf{k}^{2}-m E}} .
\end{aligned}
$$

\footnotetext{
${ }^{1}$ Note that there exists an alternative form of the dimer formalism, in which the dimer has a kinetic term (see, e.g., Ref. [21]). Such a formulation might be advantageous in the presence of a shallow bound dimer. In the following, however, we do not consider this possibility separately, since our results do not depend on a particular formulation chosen.
} 
The solution of the above equation is unique because of the presence of the ultraviolet cutoff $\Lambda$. Then, in order to ensure the independence of the observables on the cutoff, as $\Lambda \rightarrow \infty$, the three-body coupling $H_{0}(\Lambda)=\Lambda^{2} h_{0}(\Lambda) /\left(m f_{0}^{2}\right)$ should depend on $\Lambda$ in a log-periodic manner [22, 23]. As mentioned previously, the three-particle on-shell scattering amplitude can be straightforwardly obtained from the particle-dimer amplitude $\mathcal{M}$ by attaching the two-particle-dimer vertex to each of the external dimer legs.

Our study is aimed at the extraction of the finite-volume energy spectrum of a system described by the Lagrangian (4). In a finite volume, the momentum integrations in the Feynman integrals are replaced by sums over the discrete momenta. Thus, Eq. (6) in a finite volume takes the form

$$
\mathcal{M}_{L}(\mathbf{p}, \mathbf{q} ; E)=Z(\mathbf{p}, \mathbf{q} ; E)+\frac{8 \pi}{L^{3}} \sum_{\mathbf{k}}^{\Lambda} Z(\mathbf{p}, \mathbf{k} ; E) \tau_{L}(\mathbf{k} ; E) \mathcal{M}_{L}(\mathbf{k}, \mathbf{q} ; E),
$$

where $\mathbf{k}=\frac{2 \pi}{L} \mathbf{n}, \mathbf{n} \in \mathbb{Z}^{3}$ is a quantized three-momentum in a finite volume and

$$
\tau_{L}^{-1}(\mathbf{k} ; E)=k^{*} \cot \delta\left(k^{*}\right)+S\left(\mathbf{k},\left(k^{*}\right)^{2}\right)
$$

where

$$
S\left(\mathbf{k},\left(k^{*}\right)^{2}\right)=-\frac{4 \pi}{L^{3}} \sum_{\mathbf{l}} \frac{1}{\mathbf{k}^{2}+\mathbf{l}^{2}+\mathbf{k} \mathbf{l}-m E} .
$$

This sum diverges in the ultraviolet and needs to be regularized and renormalized (for the details, see Ref. [20]).

The finite-volume energy spectrum is given by the poles of the three-particle Green function which are, in its turn, determined by the poles of the quantity $\mathcal{M}_{L}$ given by Eq. (8). The latter coincide with the zeros of the determinant of the system of linear equations, which emerges from Eq. (8) after the discretization of momenta. Equating the determinant to zero finally gives the quantization condition in the particle-dimer picture one is looking for.

Now, our strategy for the analysis of data in the three-particle sector can be formulated as follows:

1. Consider first the two-particle sector, extract the phase shift $\delta(p)$ at different momenta by using the Lüscher equation. Parameterize the function $p \cot \delta(p)$ so that it fits the lattice data and does not lead to spurious poles at large momenta.

2. Fix the cutoff $\Lambda$. Truncate the partial-wave expansion (consider dimers with a spin below some fixed value). Fit the spectrum in the three-particle sector, using $h_{0}, h_{2}, \ldots$ as free parameters. Repeat this until the fit does not improve anymore by adding parameters.

3. Solve the equations in the infinite volume by using the same values of the parameters and the same cutoff $\Lambda$. Calculate different cross sections, bound-state energies, etc.

It can be shown that the proposed approach, in its range of applicability, is equivalent to the ones that were considered previously in the literature [3, 7-9]. Its advantage consists in its simplicity, which stems from the choice of the low-energy observables that should be fitted to the lattice data in the three-particle sector. While the earlier approaches, generally, have undertaken a tour de force to link the finite-volume spectrum to the three-particle $S$-matrix elements, here we propose to determine few particle-dimer couplings $h_{0}, \ldots$, that leads to much simpler equations. It remains to be seen, how advantageous this simplicity can be in the analysis of data from real simulations. 


\section{Three-particle bound state}

In Ref. [4], it has been shown that the leading finite-volume energy shift of a shallow bound state of the identical bosons in the unitary limit $a \rightarrow \infty$ is given by ${ }^{2}$

$$
\Delta E=-96.351 \frac{\kappa^{2}}{m} \mathcal{A}^{2}(\kappa L)^{-3 / 2} \exp \left(-\frac{2}{\sqrt{3}} \kappa L\right) .
$$

Here, $\kappa=\sqrt{-m E_{B}}$ is the binding momentum of the state with the energy $E_{B}$ and $\mathcal{A}$ denotes the so-called asymptotic normalization coefficient. The subleading corrections are suppressed at least by one power of $\kappa L$, or by exponentials with the argument proportional to $\kappa L$. It is assumed that the condition $\kappa L \gg 1$ holds. Our aim is to demonstrate, how does one derive the same result by using the particle-dimer formalism and to explore, whether there is a possibility to generalize this result for a finite two-body scattering length. In order to achieve this goal, we first use the Poisson summation formula and get

$$
\begin{aligned}
\mathcal{M}_{L}(\mathbf{p}, \mathbf{k} ; E) & =Z(\mathbf{p}, \mathbf{k} ; E)+8 \pi \int^{\Lambda} \frac{d^{3} \mathbf{q}}{(2 \pi)^{3}} Z(\mathbf{p}, \mathbf{q} ; E) \hat{\tau}_{L}(\mathbf{q} ; E) \mathcal{M}_{L}(\mathbf{q}, \mathbf{k} ; E) \\
\hat{\tau}_{L}(\mathbf{q} ; E) & =\frac{1+\sum_{\mathbf{n} \neq \mathbf{0}} e^{2 i \pi \mathbf{n} \hat{\mathbf{~}}}}{\tau^{-1}(\mathbf{q} ; E)+\Delta_{L}(\mathbf{q} ; E)}, \quad \Delta_{L}(\mathbf{q} ; E)=-\frac{1}{\pi L} \int d^{3} \mathbf{s} \sum_{\mathbf{n} \neq \mathbf{0}} \frac{e^{2 \pi i \mathbf{n} \mathbf{s}-i \pi \hat{\mathbf{q}} \mathbf{n}}}{\hat{\kappa}_{L}^{2}+\frac{3}{4} \hat{\mathbf{q}}^{2}+\mathbf{s}^{2}}
\end{aligned}
$$

Eliminating now the quantity $Z$ using Eq. (6), we obtain

$$
\mathcal{M}_{L}(\mathbf{p}, \mathbf{k} ; E)=\mathcal{M}(\mathbf{p}, \mathbf{k} ; E)+8 \pi \int^{\Lambda} \frac{d^{3} \mathbf{q}}{(2 \pi)^{3}} \mathcal{M}(\mathbf{p}, \mathbf{k} ; E) \delta \tau_{L}(\mathbf{q} ; E) \mathcal{M}_{L}(\mathbf{q}, \mathbf{k} ; E),
$$

where

$$
\begin{aligned}
\delta \tau_{L}(\mathbf{q} ; E) & =\hat{\tau}_{L}(\mathbf{q} ; E)-\tau(\mathbf{q} ; E) \\
& =\sum_{\mathbf{n} \neq \mathbf{0}} e^{2 i \pi \mathbf{n}} \hat{\mathbf{q}}_{\tau}(\mathbf{q} ; E)-\left(1+\sum_{\mathbf{n} \neq \mathbf{0}} e^{2 i \pi \mathbf{n} \hat{\mathbf{q}}}\right)(\tau(\mathbf{q} ; E))^{2} \Delta_{L}(\mathbf{q} ; E)+\cdots
\end{aligned}
$$

The infinite-volume amplitude $\mathcal{M}$ has a pole at the bound-state energy

$$
\mathcal{M}(\mathbf{p}, \mathbf{k} ; E)=\frac{\Psi(\mathbf{p}) \Psi(\mathbf{k})}{E-E_{B}}+\text { terms regular as } E \rightarrow E_{B},
$$

where $\Psi(\mathbf{p})$ is the infinite-volume wave function. Substituting this ansatz in Eq. (13), we finally obtain the expression for the first-order energy shift of the three-body bound state

$$
\Delta E=8 \pi \int^{\Lambda} \frac{d^{3} \mathbf{p}}{(2 \pi)^{3}}(\Psi(\mathbf{p}))^{2} \delta \tau_{L}(\mathbf{p} ; E) .
$$

\footnotetext{
${ }^{2}$ The same result has been obtained in Ref. [12] in a different formalism.
} 
Further, the energy shift can be written as

$$
\begin{aligned}
\Delta E & =\Delta E_{1}+\Delta E_{2}+\cdots, \\
\Delta E_{1} & =8 \pi \int^{\Lambda} \frac{d^{3} \mathbf{p}}{(2 \pi)^{3}} \frac{(\Psi(\mathbf{p}))^{2} \sum_{\mathbf{n} \neq \mathbf{0}} e^{2 i \pi \mathbf{n} \hat{\mathbf{p}}}}{-a^{-1}+\sqrt{\frac{3}{4} \mathbf{p}^{2}+\kappa^{2}}}, \\
\Delta E_{2} & =-8 \pi \int^{\Lambda} \frac{d^{3} \mathbf{p}}{(2 \pi)^{3}} \frac{(\Psi(\mathbf{p}))^{2}\left(1+\sum_{\mathbf{n} \neq \mathbf{0}} e^{2 i \pi \mathbf{n} \hat{p}}\right)}{\left(-a^{-1}+\sqrt{\frac{3}{4} \mathbf{p}^{2}+\kappa^{2}}\right)^{2}} \Delta_{L}(\mathbf{p}, E) .
\end{aligned}
$$

It can be shown that the integral $\Delta E_{1}$ gives the leading contribution both in the unitary limit and beyond it (see Refs. [12, 19]). Further, the leading exponential contribution to $\Delta E_{1}$ emerges from the terms with $|\mathbf{n}|=1$. Consequently, the whole calculation boils down to substituting the exact infinite-volume wave function in the above integral and evaluating it explicitly.

In the unitary limit, with $\Lambda \rightarrow \infty$ and $H_{0}(\Lambda) \rightarrow 0$, the analytic form of the infinite-volume wave function was given by Minlos and Faddeev, see Ref. [24]. This solution should be properly normalized. Since the kernel of the integral equation depends on the energy $E$, the normalization condition can no more be written down in a trivial form and contains the terms that depend on the interaction (see Ref. [19] for more details). At the end of the day, substituting the normalized wave function into the expression for the energy shift and evaluating the integral, we arrive at Eq. (11) with $\mathcal{A}=1$.

This, however, is not the end of the story, because the Bethe-Salpeter equation without the cutoff and the three-body term $H_{0}$ is known to be ill-defined mathematically. Restoring both, we do not have an analytic solution any more. The key observation is, however, that the equation is modified only at very high momenta (comparable to the cutoff scale $\Lambda$ ). The wave function at low momenta is also modified, because the whole wave function should be normalized to unity, where the normalization integral runs over the low as well as high momenta. To summarize, at low momenta the true wave function must be given as

$$
\Psi(\mathbf{p})=\mathcal{A} \Psi_{0}(\mathbf{p}),
$$

where $\Psi_{0}(\mathbf{p})$ is the Minlos-Faddeev wave function and $\mathcal{A}$ is the particle-dimer asymptotic normalization coefficient, which can be determined numerically in the infinite volume. One can now use this form in the expression of the energy shift, because there only the low-momentum region matters. Then, finally, Eq. (11) is reproduced. This derivation, however, sheds light on the physical meaning of the asymptotic normalization coefficient $\mathcal{A}$ as well: this sole quantity encodes in it the whole effect of the three-body short range interactions carried by the couplings $h_{i}$. It can be rigorously shown that $\mathcal{A} \rightarrow 1$ as $\Lambda \rightarrow \infty$, if there are no derivative interactions in the particle-dimer sector and, in general, is different from unity, if these are present.

Finally, the particle-dimer formalism, described above, allows one to go beyond the unitary limit, considered in Refs. [4, 12]. The leading-order energy shift in this case is given by

$$
\Delta E \propto \int^{\Lambda} \frac{d^{3} \mathbf{p}}{(2 \pi)^{3}} \frac{(\Psi(\mathbf{p}))^{2} e^{2 i \pi \mathbf{n} \hat{\mathbf{p}}}}{-a^{-1}+\sqrt{\frac{3}{4} \mathbf{p}^{2}+\kappa^{2}}}+\cdots, \quad|\mathbf{n}|=1
$$

It can be shown that the wave function $\Psi(\mathbf{p})$ is regular near the origin (more precisely, the singularities of $\Psi(\mathbf{p})$ are located much farther from the origin than the singularities of the denominator). This means 
that the singularities of $\Psi(\mathbf{p})$ do not contribute to the large- $L$ behavior of the energy shift at leading order and hence, at this order, $\Psi(\mathbf{p})$ can be replaced by a constant. Performing the angular integration, we arrive at the following result

$$
\Delta E \propto \frac{1}{L} \int_{-\infty}^{\infty} \frac{p d p}{2 \pi i} \frac{e^{i p L}\left(a^{-1}+\sqrt{\frac{3}{4} p^{2}+\kappa^{2}}\right)}{\frac{3}{4} p^{2}+\kappa^{2}-a^{-2}} .
$$

Note that the quantity $\kappa^{2}-a^{-2}$ is always positive, if a bound state of a particle and a bound dimer is considered (recall that $\kappa_{2}=a^{-1}$ is the binding momentum of the dimer in the unitary limit).

Now, if $\kappa^{2}-a^{-2} \ll \kappa^{2}$ that corresponds to the case of a shallow bound state of a particle and a deeply bound dimer, the singularity at $p= \pm i \sqrt{\frac{4}{3}\left(\kappa^{2}-a^{-2}\right)}$ is dominant, and the singularity arising from the square root (cut) can be neglected. Performing the Cauchy integration, we get

$$
\Delta E \propto \frac{1}{L} \exp \left(-\frac{2}{\sqrt{3}} \sqrt{\kappa^{2}-a^{-2}} L\right) .
$$

In other words, we reproduce Lüscher's original result for a two particle (particle-dimer) bound state. In the opposite limit $\kappa^{2} \gg a^{-2}$, which corresponds to a shallow bound state of three particles, the first term in Eq. (20) is very small and the energy shift is dominated by the second term (here, the term "shallow bound state" means that $\kappa \ll \Lambda$ and/or $\kappa \ll r^{-1}$, where $r$ denotes the interaction range). It is straightforward to see that, in this case,

$$
\Delta E \propto \frac{1}{L^{3 / 2}} \exp \left(-\frac{2}{\sqrt{3}} \kappa L\right) .
$$

In other words, the result of Ref. [4] is reproduced in this limit.

\section{Conclusions}

1. We have proposed a framework for the extraction of hadronic observables from the lattice spectrum in the three-particle sector. The approach is based on the particle-dimer formulation of the non-relativistic effective field theory in a finite volume. It is proposed to fit the three-particle (particle-dimer) couplings to the lattice spectrum and then calculate the hadronic observables by using the NREFT machinery in the infinite volume.

2. It is shown that the approach - in its range of applicability - is equivalent to other approaches known in the literature. Yet, it is much simpler due to a convenient choice of the observables which are to be determined from the lattice data.

3. We have demonstrated the potential of the approach by calculating the finite-volume energy shift of the three-body bound state. Our approach allows one not only to reproduce the known result, but to go - with a surprising ease - beyond the approximations used in the original derivation.

Acknowledgments. The authors would like to thank R. Briceno, Z. Davoudi, M. Döring, E. Epelbaum, M. Hansen, D. Lee, T. Luu, M. Mai, U.-G. Meißner and S. Sharpe for useful discussions.

We acknowledge support from the DFG through funds provided to the Sino-German CRC 110 "Symmetries and the Emergence of Structure in QCD" and the CRC 1245 "Nuclei: From Fundamental Interactions to Structure and Stars" as well as the BMBF under contract 05P15RDFN1. This research is also supported in part by Volkswagenstiftung under contract no. 86260 and by Shota Rustaveli National Science Foundation (SRNSF), grant no. DI-2016-26. 


\section{References}

[1] M. Lüscher, Nucl. Phys. B354, 531 (1991)

[2] L. Lellouch, M. Lüscher, Commun. Math. Phys. 219, 31 (2001), hep-lat/0003023

[3] K. Polejaeva, A. Rusetsky, Eur. Phys. J. A48, 67 (2012), 1203. 1241

[4] U.G. Meißner, G. Ríos, A. Rusetsky, Phys. Rev. Lett. 114, 091602 (2015), [Erratum: Phys. Rev. Lett. 117, 069902(2016)], 1412.4969

[5] P. Guo, Phys. Rev. D95, 054508 (2017), 1607.03184

[6] P. Guo, V. Gasparian (2017), 1701.00438

[7] R.A. Briceno, Z. Davoudi, Phys. Rev. D87, 094507 (2013), 1212 . 3398

[8] M.T. Hansen, S.R. Sharpe, Phys. Rev. D90, 116003 (2014), 1408. 5933

[9] M.T. Hansen, S.R. Sharpe, Phys. Rev. D92, 114509 (2015), 1504.04248

[10] M.T. Hansen, S.R. Sharpe, Phys. Rev. D93, 014506 (2016), 1509. 07929

[11] M.T. Hansen, S.R. Sharpe, Phys. Rev. D93, 096006 (2016), [Erratum: Phys. Rev. D96, 039901 (2017)], 1602.00324

[12] M.T. Hansen, S.R. Sharpe, Phys. Rev. D95, 034501 (2017), 1609.04317

[13] S. Kreuzer, H.W. Hammer, Phys. Lett. B694, 424 (2011), 1008.4499

[14] S. Kreuzer, H.W. Hammer, Eur. Phys. J. A43, 229 (2010), 0910. 2191

[15] S. Kreuzer, H.W. Hammer, Phys. Lett. B673, 260 (2009), 0811.0159

[16] S. Kreuzer, H.W. Grießhammer, Eur. Phys. J. A48, 93 (2012), 1205.0277

[17] P. Guo, V. Gasparian (2017), 1709.08255

[18] S.R. Sharpe, Phys. Rev. D96, 054515 (2017), 1707.04279

[19] H.W. Hammer, J.Y. Pang, A. Rusetsky, JHEP 09, 109 (2017), 1706.07700

[20] H.W. Hammer, J.Y. Pang, A. Rusetsky (2017), 1707.02176

[21] D.B. Kaplan, Nucl. Phys. B494, 471 (1997), nucl-th/9610052

[22] P.F. Bedaque, H.W. Hammer, U. van Kolck, Phys. Rev. Lett. 82, 463 (1999), nucl-th/9809025

[23] P.F. Bedaque, H.W. Hammer, U. van Kolck, Nucl. Phys. A646, 444 (1999), nucl-th/9811046

[24] L.D. Faddeev, R.A. Minlos, Sov. Phys. JETP 14, 1315 (1962), [Zh. Eksp. Teor. Fiz. 41, 1850 (1961)] 